\title{
Artroscopia bilateral de rodilla en un solo tiempo quirúrgico, ¿es una opción segura?
}

\author{
Mariano García Bistolfi, Juan Zícaro, Tomás Gorodischer, Carlos Yacuzzi, Matías Costa Paz \\ Sector Rodilla, Servicio de Ortopedia y Traumatología, Hospital Italiano de Buenos Aires, Ciudad Autónoma de Buenos Aires, Argentina
}

\section{RESUMEN}

Introducción: La artroscopia bilateral de rodilla en un solo tiempo quirúrgico permite cursar un solo posoperatorio y una única rehabilitación. El objetivo de este estudio fue evaluar los resultados clínico-funcionales y el tiempo hasta el retorno laboral y deportivo en una serie de pacientes sometidos a una artroscopia bilateral en un solo tiempo quirúrgico. Materiales y Métodos: Se evaluó a una serie retrospectiva de pacientes desde abril de 2016 hasta abril de 2019, que fueron sometidos a una artroscopia bilateral de rodilla en un solo tiempo quirúrgico. Se analizaron los resultados clínico-funcionales a corto y mediano plazo, y el tiempo para el retorno laboral y deportivo. Resultados: La edad promedio fue de 41 años (rango 18-63). El seguimiento promedio fue de 18 meses (rango 6-37). Los tiempos de anestesia y quirúrgico promedio fueron 105 min (rango 60-170) y 85 min (rango 50-150), respectivamente. El tiempo promedio para el retorno laboral fue de 2 meses (rango 1-5). Todos los pacientes recuperaron el rango completo de movilidad articular. Conclusiones: Si bien se han obtenido buenos resultados clínicos con la artroscopia bilateral de rodilla en un solo tiempo, en pacientes seleccionados, no se pueden establecer comparaciones ni conclusiones relevantes debido a la baja casuística y a la gran diversidad de las cirugías realizadas. La principal ventaja radicaría en evitar procedimientos en dos tiempos quirúrgicos, lo que implicaría dos operaciones, dos anestesias y dos programas de rehabilitación diferentes.

Palabras clave: Artroscopia bilateral; artroscopia en un tiempo quirúrgico; artroscopia de rodilla.

Nivel de Evidencia: IV

\section{Is Single-Stage Bilateral Knee Arthroscopy a Safe Option?}

\section{ABSTRACT}

Introduction: Single-stage bilateral arthroscopic surgery allows the patient to undergo a single postoperative and rehabilitation period. The aim of this article was to evaluate the functional-clinical outcomes and time to return to work and sports in a series of patients who had undergone single-stage bilateral arthroscopy. Materials and Methods: We evaluated a retrospective series of patients who had undergone single-stage bilateral knee arthroscopy from April 2016 to April 2019. Short- and medium-term clinical-functional outcomes, and time to return to work and sports were analyzed. Results: The average age of the patients was 41 years (range $18-63$ ), with an average follow-up of 18 months (6-37). The average anesthesia time was 105 minutes (range $60-170$ ) and the average surgical time was 85 minutes (50 to 150). The average time to return to work was 2 months (range 1-5). Joint range of motion was fully recovered in all patients. Conclusion: Although single-stage bilateral arthroscopy has shown good clinical outcomes in selected patients, no relevant comparisons or conclusions can be established due to the low casuistry and the great diversity of the surgeries performed. The main advantage would be in avoiding procedures in two surgical stages, which would imply two operations, twice the anesthesia and two different rehabilitation programs.

Keywords: Bilateral arthroscopy; single-stage arthroscopy; knee arthroscopy.

Level of Evidence: IV

Recibido el 18-3-2020. Aceptado luego de la evaluación el 30-9-2020 • Dr. MARIANO GARCíA BISTOLFI • mariano.garciabistolfi@ hospitalitaliano.org.ar ID https://orcid.org/0000-0002-5444-6927 Cómo citar este artículo: García Bistolfi M, Zícaro J, Gorodischer T, Yacuzzi C, Costa Paz M. Artroscopia bilateral de rodilla en un solo tiempo quirúrgico, ¿es una opción segura? Rev Asoc Argent Ortop Traumatol 2021;86(3):309-315. https://doi.org/10.15417/issn.1852-7434.2021.86.3.1077 


\section{INTRODUCCIÓN}

Cuando un paciente requiere cirugía artroscópica en ambas rodillas, el procedimiento se puede realizar en un solo acto quirúrgico o bien planificarlo en dos tiempos. La artroscopia bilateral en un solo tiempo puede llevarse a cabo en forma secuencial, con un único equipo médico, ${ }^{1-3} \mathrm{o}$ simultáneamente con dos equipos diferentes. ${ }^{4.5}$ Esto le permite al paciente cursar un solo posoperatorio y una única rehabilitación. A su vez, el tiempo hasta el retorno laboral y deportivo, si bien depende del tipo de cirugía, suele ser similar al de las cirugías en dos tiempos quirúrgicos diferentes. ${ }^{1,4,5}$

Los procedimientos bilaterales en un solo tiempo implican un menor costo que aquellos que se realizan en dos tiempos. En distintas series, se comunica una reducción del gasto económico de entre el $29 \%$ y el $64 \%$ comparado con el de la cirugía en dos tiempos y que se atribuye principalmente a los cargos quirúrgicos y de cuidados hospitalarios..$^{1-3,5}$

En ninguna de las series reportadas, se observó un mayor riesgo de complicaciones entre las cirugías en uno o dos tiempos quirúrgicos. ${ }^{1-3,5}$

En la actualidad, son escasos los artículos publicados que informan los resultados clínicos o de retorno laboral tras la cirugía artroscópica bilateral.

El objetivo principal de este estudio fue evaluar una serie de pacientes sometidos a una artroscopia bilateral de rodilla en un solo tiempo, desde el punto de vista clínico y funcional, así como también analizar el tiempo hasta el retorno laboral y deportivo.

\section{MATERIALES Y MÉTODOS}

Se analizó una serie de casos, de manera retrospectiva, en la que se incluyeron 11 pacientes sometidos a cirugía artroscópica de ambas rodillas en un solo tiempo quirúrgico, ya sea en forma secuencial o simultánea, entre abril de 2016 y abril de 2019. El seguimiento mínimo fue de seis meses.

Todos los procedimientos se llevaron a cabo en el Servicio de Ortopedia y Traumatología de nuestro hospital. La búsqueda se realizó en la base de datos de historias clínicas electrónicas de la institución.

Se registraron los siguientes datos: tipo de procedimiento quirúrgico, riesgo prequirúrgico mediante la clasificación de la American Society of Anaesthesiologists (ASA), ${ }^{6}$ tiempo de anestesia y tiempo quirúrgico.

Se evaluó el dolor antes y después de la cirugía, mediante la escala analógica visual y se determinó el grado de satisfacción posoperatorio con la escala de Likert. ${ }^{7}$ El rango de movilidad articular se determinó con un goniómetro en la última consulta. Se consideró como completo en el rango de $0-10^{\circ}$ de extensión y $>120^{\circ}$ de flexión.

Se analizó el tiempo hasta la reinserción laboral y el retorno a la práctica deportiva. Se registraron las complicaciones quirúrgicas según la clasificación de Dindo-Clavien. ${ }^{6}$

\section{Técnica quirúrgica}

Se ubicó al paciente en decúbito supino. Las cirugías se realizaron bajo anestesia general y locorregional. Ambos miembros inferiores se colocaron dentro del mismo campo estéril. Las operaciones estuvieron a cargo de cirujanos especialistas en artroscopia de rodilla. Contamos con una sola torre de artroscopia y dos equipos quirúrgicos, por lo que, en aquellas cirugías solo de tiempo artroscópico, los procedimientos se realizaron de manera secuencial, mientras que, en aquellas con tiempo abierto, cuando uno de los equipos comenzó con el tiempo artroscópico en una rodilla, el otro inició el tiempo abierto en la otra. El manguito hemostático (en los casos de cirugía del ligamento cruzado anterior) se utilizó únicamente para el tiempo artroscópico (Figura).

En la Tabla 1, se resumen los procedimientos quirúrgicos. Respecto a la plástica del ligamento cruzado anterior, en todos los casos, se efectuó una técnica con injerto de isquiotibiales, fijación cortical con botón en el fémur y tornillo interferencial en la tibia.?

Los protocolos de rehabilitación fueron individualizados según el tipo de cirugía. 


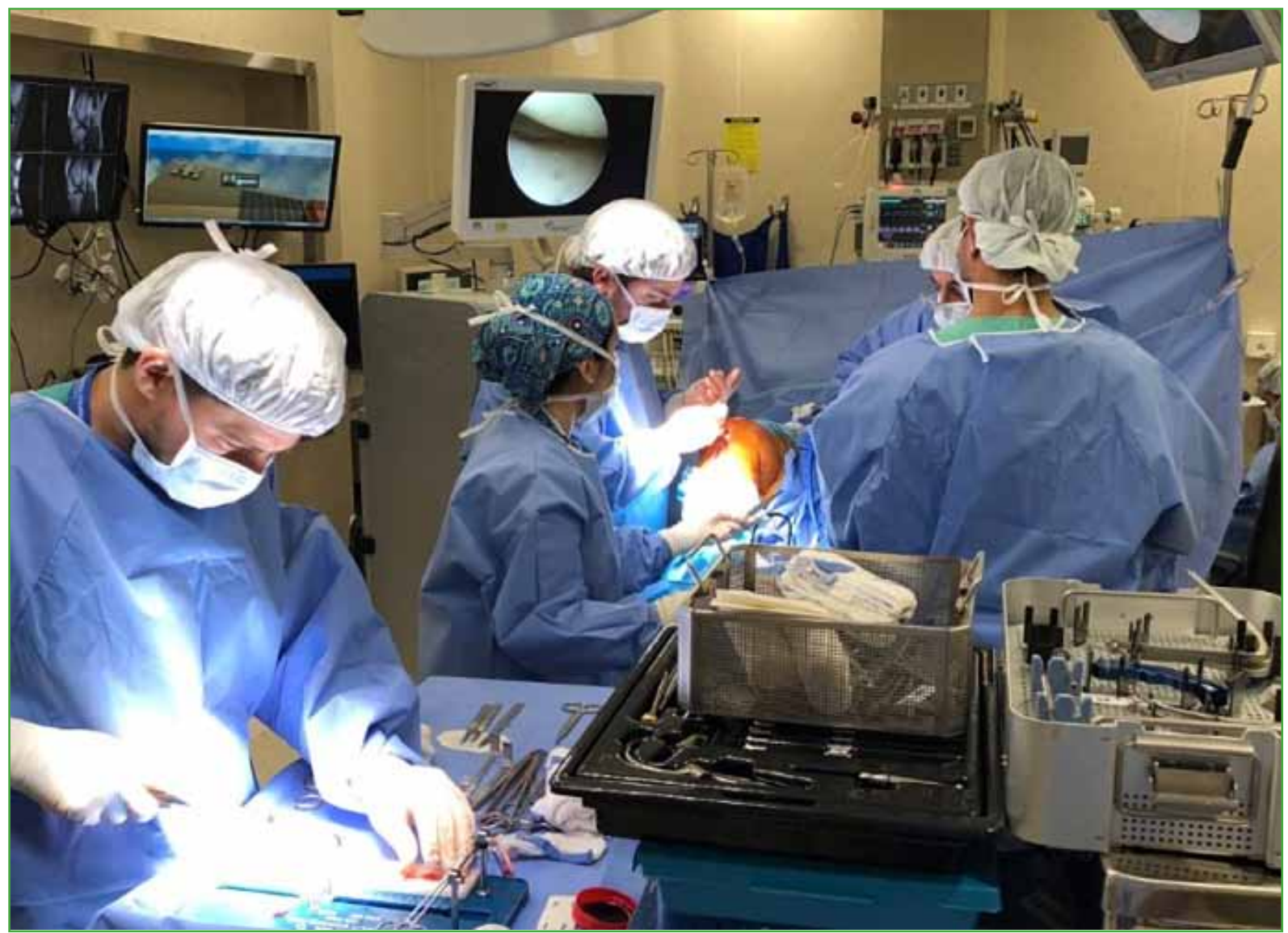

Figura. Cirugía de reconstrucción del ligamento cruzado anterior. En la rodilla derecha, se finalizó el tiempo artroscópico y se fijó el injerto. En la rodilla izquierda, se tomó el injerto durante el tiempo artroscópico de la derecha (en preparación en la mesa quirúrgica) y se está avanzando con el tiempo artroscópico.

\section{RESULTADOS}

Se evaluó a cinco mujeres y seis hombres, con un promedio de edad de 41 años (rango 18-63) y un seguimiento promedio de 18 meses (rango 6-37). El riesgo quirúrgico era ASA 1 (6 pacientes), ASA 2 (4 pacientes) y ASA 3 (1 caso). El tiempo promedio de anestesia fue de 105 min (rango 60-170). El tiempo promedio de cirugía fue de 85 $\min$ (rango 50-150). Siete procedimientos fueron ambulatorios y cuatro, con internación: tres ligamentos cruzados anteriores y una necrosis ósea avascular (Tablas 1,2 y 3 ).

La media de dolor según la escala analógica visual fue de 7/10 (rango de 3/10 a 10/10) antes de la intervención y de 2/10 (rango de 0/10 a 6/10) después. Según la escala de satisfacción de Likert, nueve pacientes refirieron estar "muy satisfechos" y dos "satisfechos" con los resultados posoperatorios. Ante la pregunta "¿se volvería a someter al mismo procedimiento bilateral en un tiempo?", todos respondieron que sí.

En la última evaluación, todos habían recuperado el rango de movilidad articular.

Los tiempos de reinserción laboral y deportiva se describen en las Tablas 1 y 2.

En el posoperatorio inmediato, se registraron dos complicaciones menores: un paciente con dolor persistente y otro con hemartrosis.

\section{DISCUSIÓN}

Independientemente de la enfermedad de base, se han logrado resultados satisfactorios con la artroscopia bilateral de rodilla en un solo tiempo quirúrgico. ${ }^{1-3,5}$ Larson y cols. no hallaron diferencias estadísticamente significativas para las tasas de complicaciones, las pruebas de inestabilidad o los resultados funcionales entre pacientes con insuficiencia del ligamento cruzado anterior bilateral operados en un solo tiempo quirúrgico y aquellos operados en dos tiempos. ${ }^{2}$ 
Tabla 1. Resumen de los procedimientos quirúrgicos

\begin{tabular}{|c|c|c|c|c|c|c|}
\hline $\begin{array}{l}\text { Pa- } \\
\text { ciente }\end{array}$ & Diagnóstico & $\begin{array}{c}\text { Tratamiento artroscópico } \\
\text { bilateral }\end{array}$ & $\begin{array}{l}\text { Tiempo de } \\
\text { anestesia }\end{array}$ & $\begin{array}{l}\text { Tiempo } \\
\text { quirúrgico }\end{array}$ & $\begin{array}{l}\text { Tiempo hasta el } \\
\text { retorno laboral } \\
\text { (semanas) }\end{array}$ & $\begin{array}{l}\text { Tiempo hasta el } \\
\text { retorno al deporte } \\
\text { (semanas) }\end{array}$ \\
\hline 1 & LOC en rótula & $\begin{array}{l}\text { Microperforaciones } \\
\text { + PRP }\end{array}$ & $60 \mathrm{~min}$ & $50 \mathrm{~min}$ & 20 & 12 \\
\hline 2 & $\begin{array}{l}\text { Rotura de LCA } \\
\quad+\text { SMI }\end{array}$ & $\begin{array}{l}\text { Reconstrucción del LCA } \\
+ \text { sutura meniscal }\end{array}$ & $170 \mathrm{~min}$ & $140 \mathrm{~min}$ & 16 & 32 \\
\hline 3 & $\begin{array}{l}\text { LOC en cóndilo } \\
\text { femoral + SMI }\end{array}$ & $\begin{aligned} & \text { Microperforaciones } \\
+ & \text { menisectomía parcial }\end{aligned}$ & $70 \mathrm{~min}$ & $50 \mathrm{~min}$ & 12 & 12 \\
\hline 4 & $\begin{array}{l}\text { LOC en cóndilo } \\
\text { femoral + SMI }\end{array}$ & $\begin{array}{l}\text { Microperforaciones } \\
\text { en cóndilo femoral } \\
+ \text { menisectomía parcial }\end{array}$ & $75 \mathrm{~min}$ & $60 \mathrm{~min}$ & 8 & 8 \\
\hline 5 & $\begin{array}{c}\text { Rotura de LCA } \\
+ \text { SMI }\end{array}$ & $\begin{array}{l}\text { Reconstrucción del LCA } \\
\text { + menisectomía parcial }\end{array}$ & $110 \mathrm{~min}$ & $75 \mathrm{~min}$ & 8 & 32 \\
\hline 6 & $\begin{array}{l}\text { Rotura de LCA } \\
\quad+\text { SMI }\end{array}$ & $\begin{array}{l}\text { Reconstrucción del LCA } \\
+ \text { menisectomía parcial }\end{array}$ & $150 \mathrm{~min}$ & $105 \mathrm{~min}$ & 8 & 36 \\
\hline 7 & SMI & Menisectomía parcial & $90 \mathrm{~min}$ & $80 \mathrm{~min}$ & 1 & 6 \\
\hline 8 & $\begin{array}{l}\text { Necrosis avascular } \\
\text { en cóndilo femoral }\end{array}$ & $\begin{array}{l}\text { Microperforaciones en } \\
\text { cóndilo femoral + con- } \\
\text { centrado de médula ósea }\end{array}$ & $85 \mathrm{~min}$ & $75 \mathrm{~min}$ & No trabaja & 8 \\
\hline 9 & LOC de tróclea & $\begin{array}{l}\text { Mosaicoplastia + con- } \\
\text { centrado de médula ósea }\end{array}$ & $165 \mathrm{~min}$ & $150 \mathrm{~min}$ & 8 & 12 \\
\hline 10 & SMI & Menisectomía parcial & $80 \mathrm{~min}$ & $60 \mathrm{~min}$ & 3 & 8 \\
\hline 11 & LOC en rótula & Mosaicoplastia & $110 \mathrm{~min}$ & $90 \mathrm{~min}$ & 8 & 12 \\
\hline
\end{tabular}

Tabla 2. Promedio de los tiempos de anestesia, quirúrgico, hasta el retorno laboral y deportivo

\begin{tabular}{|lcccc}
\hline $\mathbf{n}=\mathbf{1 1}$ & Media & DE & Mínimo & Máximo \\
\hline Tiempo de anestesia (min) & 106 & \pm 38.38 & 63 & 169 \\
Tiempo de cirugía (min) & 88 & \pm 42.02 & 49 & 181 \\
Tiempo hasta el retorno laboral (semanas) & 9 & \pm 5.61 & 1 & 20 \\
Tiempo hasta el retorno al deporte (semanas) & 16 & \pm 11.26 & 6 & 36
\end{tabular}

$\mathrm{DE}$ = desviación estándar.

Habitualmente la artroscopia de rodilla es de manejo ambulatorio; sin embargo, en los procedimientos bilaterales en una sola etapa, no hay un consenso claro. ${ }^{2,3,8,9}$ Algunos autores recomiendan, al menos, un día de internación, debido a la dificultad para deambular y la menor movilidad de estos pacientes, ${ }^{2,3,8}$ mientras que otros prefieren un manejo ambulatorio. ${ }^{3,9}$ En nuestra serie, el manejo de los pacientes sometidos a una reconstrucción del ligamento cruzado anterior bilateral incluyó internación debido al bloqueo regional de ambos miembros inferiores. El paciente restante quedó internado a causa del alto riesgo quirúrgico (ASA 3) por antecedente de trasplante pulmonar. El resto de las cirugías fueron ambulatorias, ya que se realizaron con anestesia local y general. 
Tabla 3. Cantidad de cirugías ambulatoria y con internación, y ASA

\begin{tabular}{|l|c|}
\hline Tipo de intervención & Total \\
\hline Ambulatoria & 7 \\
\hline No ambulatoria & 4 \\
\hline & $\mathrm{n}=11$ \\
\hline ASA & \\
\hline 1 & 6 \\
\hline 2 & 4 \\
\hline 3 & 1 \\
\hline
\end{tabular}

Un factor importante por tener en cuenta al realizar una artroscopia bilateral en un único tiempo quirúrgico es la comprensión y el deseo del paciente, ya que esto es de suma importancia para la buena evolución y la rehabilitación. La falta de convicción puede ser un factor limitante a la hora de efectuar una artroscopia bilateral en un solo tiempo quirúrgico. ${ }^{4,5}$

El retorno a la actividad laboral y deportiva luego de una artroscopia simple depende no solo del diagnóstico inicial y del tipo de tratamiento propuesto, sino también de factores intraoperatorios, como el tratamiento del cartílago articular, la necesidad de osteotomías o reconstrucciones ligamentarias que incluso podrían extender el tiempo hasta el retorno laboral a más de tres meses. En la serie publicada por Jari y cols., ${ }^{1}$ no se hallaron diferencias significativas en el tiempo hasta la reinserción laboral en pacientes con cirugía en un solo tiempo quirúrgico y aquellos con una operación en etapas.

En la Tabla 4, se comparan la media del tiempo hasta el retorno laboral y deportivo según el tipo de cirugía cuando es unilateral o bilateral. Si bien los lapsos pueden ser levemente mayores al operar ambas rodillas en un tiempo, el paciente no requiere un segundo procedimiento, pero aumentaría la ausencia laboral y retrasaría el retorno al deporte. La ventaja de operar ambas rodillas en un tiempo radica en cursar un único posoperatorio.

Si bien el lapso hasta el retorno laboral fue similar al de otras series publicadas, ${ }^{1,4}$ debemos destacar que depende, en gran medida, del tipo de intervención y del tipo de actividad a la que se dedica cada paciente.

La tasa de complicaciones publicada para las artroscopias simples y las bilaterales realizadas en un mismo tiempo quirúrgico son similares, entre el 1\% y el 6\%. Las complicaciones más frecuentes en las cirugías bilaterales son dolor y hemartrosis, ${ }^{8-11}$ lo que coincide con las dos complicaciones observadas en nuestra serie.

Tabla 4. Comparación de la media del tiempo hasta el retorno laboral y deportivo en cirugías unilaterales y bilaterales en un tiempo quirúrgico, según cada patología, expresado en semanas

\begin{tabular}{|l|c|c|c|c|}
\hline & \multicolumn{2}{|c|}{ Unilateral } & \multicolumn{2}{|c|}{ Bilateral } \\
\hline & $\begin{array}{c}\text { Retorno } \\
\text { laboral }\end{array}$ & $\begin{array}{c}\text { Retorno } \\
\text { al deporte }\end{array}$ & $\begin{array}{c}\text { Retorno } \\
\text { laboral }\end{array}$ & $\begin{array}{c}\text { Retorno } \\
\text { al deporte }\end{array}$ \\
\hline Plástica de ligamento cruzado anterior & 8 & 32 & 11 & 32 \\
\hline Menisectomía parcial & 2 & 6 & 2 & 7 \\
\hline Mosaicoplastia & 7 & 12 & 8 & 12 \\
\hline Microperforaciones & 5 & 8 & 10 & 9 \\
\hline
\end{tabular}


Varias publicaciones señalan que la artroscopia bilateral en un único tiempo quirúrgico representa un menor costo hospitalario. ${ }^{2,5,12}$ En la serie publicada por Sajovic y cols. que compara los costos ocasionados por la reconstrucción bilateral del ligamento cruzado anterior en un solo tiempo y en dos tiempos, el ahorro total es de 2925 euros. ${ }^{4}$ En su serie retrospectiva, Larson y cols. informan un ahorro de más de 3750 dólares estadounidenses y atribuyen el mayor porcentaje de esta cifra a la cirugía ambulatoria y a la posibilidad de realizar una única rehabilitación posoperatoria. ${ }^{2}$

En nuestra institución, se compararon los costos de insumos de quirófano, y también los quirúrgicos, de rehabilitación y el tiempo de internación para la artroscopia bilateral de rodilla, el monto ahorrado para el sistema de salud fue de 750 dólares estadounidenses. ${ }^{12}$

La principal limitación de nuestro estudio es que, al tratarse de una serie de casos retrospectiva, sin grupo de control, con una gran heterogeneidad en los procedimientos quirúrgicos y una baja casuística, no es posible establecer comparaciones ni conclusiones relevantes. De todas maneras, se trata de un tema con escasos reportes en la bibliografía internacional y sin publicaciones en la bibliografía nacional, que aporta sobre la seguridad y los resultados de una artroscopia bilateral de rodilla en un solo tiempo quirúrgico.

\section{CONCLUSIONES}

Si bien con la artroscopia bilateral de rodilla en un solo tiempo se han logrado buenos resultados desde el punto de vista clínico y es un procedimiento costo-efectivo en pacientes seleccionados, no se pueden establecer comparaciones ni conclusiones relevantes debido a la baja casuística y a la gran diversidad de las cirugías realizadas en este estudio. La principal ventaja radicaría en evitar procedimientos en dos tiempos, lo que implicaría dos operaciones, dos anestesias y dos programas de rehabilitación diferentes.

Conflicto de intereses: Los autores no declaran conflictos de intereses.

\section{BIBLIOGRAFÍA}

1. Jari S, Shelbourne KD. Simultaneous bilateral anterior cruciate ligament reconstruction. Am J Sports Med 2002;30(6):891-5. https://doi.org/10.1177/03635465020300062201

2. Larson CM, Fischer DA, Smith JP, Boyd JL. Bilateral anterior cruciate ligament reconstruction as a single procedure: evaluation of cost and early functional results. Am J Sports Med 2004;32(1):197-200. https://doi.org/10.1177/0363546503260721

3. Vaishya R, Esin ARI, Agarwal AK, Vijay V. Bilateral simultaneous anterior cruciate ligament reconstruction: A case series and review of the literature. J Clin Orthop Trauma 2019;10(3):576-80. https://doi.org/10.1016/j.jcot.2018.08.013

4. Sajovic M, Demsar S. One-stage bilateral anterior cruciate ligament reconstruction with use of hamstring tendon autografts: a case report. Knee Surg Sports Traumatol Arthrosc 2007;15(9):1114-5. https://doi.org/10.1007/s00167-007-0286-z

5. Saithna A, Arbuthnot J, Carey-Smith R, Spalding T. Simultaneous bilateral anterior cruciate ligament reconstruction: a safe option. Knee Surg Sports Traumatol Arthrosc 2010;18(8):1071-4. https://doi.org/10.1007/s00167-009-0971-1

6. Daabiss M. American Society of Anaesthesiologists physical status classification. Indian J Anaesth 2011;55(2):1115. https://doi.org/10.4103/0019-5049.79879

7. Sullivan GM, Artino AR. Analyzing and interpreting data from Likert-type scales. J Grad Med Educ 2013;5(4):5412. https://doi.org/10.4300/JGME-5-4-18 
8. Allum RL, Ribbans WJ. Day case arthroscopy and arthroscopic surgery of the knee. Ann R Coll Surg Engl 1987;69(5):225-6. PMID: 3674684

9. Small NC. Complications in arthroscopic surgery performed by experienced arthroscopists. Arthroscopy 1988;4(3):215-21. https://doi.org/10.1016/s0749-8063(88)80030-6

10. Paisley AM, Paterson-Brown S. Minor morbidity after day-case surgery. Scott Med J 1998;43(4):105-6. PMID: 9757498

11. Kulkarni A, Brooks S, Pynsent PB. Is bilateral knee arthroscopy suitable as a day-case procedure? Knee 2003;10(3):287-9. https://doi.org/10.1016/s0968-0160(02)00146-1

12. Llano L, Yacuzzi C, Zicaro J, Costa Paz M. Reconstrucción artroscópica del ligamento cruzado anterior bilateral en un tiempo quirúrgico: reporte de casos y revisión bibliográfica. Artroscopia (Buenos Aires) 2019;26(4):127-30. Disponible en:

https://www.revistaartroscopia.com/ediciones-anteriores/images/26_04/Reconstruccin_Artroscpica_del_Ligamento_Cruzado.pdf. 